\title{
Genome-Wide Identification of MYB Gene Family Reveals Their Potential Functions in Cadmium Stress Response and the Regulation of Cannabinoid
}

Biosynthesis in Hemp (Cannabis sativa L.)

Ming Yin1,2\#, Gen Pan1,2\#, Jie Tao1,2, Lining Zhao1,2, Zheng Li1,2, Hui Jiang1,2, Huijuan Tang1,2, Li Chang1,2, Jianjun Li1,2, Anguo Chen1,2, Yong Deng ${ }_{1,2}$, Cuiping Zhang1,2, Defang Li1,2*, and Siqi Huang 1,2* $^{*}$ 1 Institute of Bast Fiber Crops, Chinese Academy of Agricultural Sciences, Changsha 410205 China, 2 Key Laboratory of the Biology and Process of Bast Fiber Crops, Ministry of Agriculture and Rural Affairs, Changsha, China

Corresponding author: SQH, huangsiqi@caas.cn; DFL, chinakenaf@126.com

\#: This authors contributed equally to this work

\section{Abstract}

Backgroud: Heavy metal pollutant, Cadmium (Cd), is an inorganic pollutant in China. Hemp (Cannabis sativa L.) is an important fiber crop to remediate heavy metal-contaminated soils. The MYB family is one of the largest and most important gene families that influences plant growth and produce secondary metabolites.

Results: $115 C S M Y B$ genes were identified in the hemp genome. The 1R-MYB subfamily had 17 genes, the 2R-MYB subfamily 88 genes, and the 3R-MYB subfamily 8 genes. The synteny analysis of the CsMYB genes indicated that there were 3 pairs of tandem repeats, 21 pairs of segmental duplication, $6 C S M Y B$ genes present before species differentiation, and 60 genes belonging to the hemp-specific $C s M Y B$ genes. 7 CsMYB genes were identified to influence the response to $\mathrm{Cd}$ stress by combining the transcriptome data of two hemp species under $\mathrm{Cd}$ stress. Based on the changes in the Cannabinoid content of hemp under Cd stress, the expression of different CSMYB genes in hemp with high and low cannabidiol (CBD) contents, and tissue-specific expression, it was inferred that CsMYB024 may be affected by $\mathrm{Cd}$ stress and mediate the CBD synthesis pathway.

Conclusions: This study provides a comprehensive analysis of the hemp MYB family. These results should be helpful in understanding of their potential functions in Cd-Stress Response and the Regulation of Cannabinoid Biosynthesis in Hemp. In addition, lays the foundation for the further study of biological functions of CsMYBs in hemp.

Keywords: hemp; cadmium stress; CsMYB; cannabinoid biosynthesis

\section{Introduction}


Plants are subjected to various abiotic stresses, which seriously affect plant growth and development [1]. Heavy metal pollution is a common abiotic stressor, with Cd ranking first among inorganic pollutants in China and being the most serious pollutant of arable land [2]. When plants are exposed to heavy metals, many genes change their expression and produce secondary metabolites that defend against the external stresses. Identifying key genes that respond to abiotic stresses can lay the foundation for breeding highly resistant plants [3-5]. Transcription factors are a class of proteins that bind to specific sequences upstream of gene sequences and regulate biological growth by altering gene expression. The identification of key transcription factors and their functions is an important method used for studying plant growth and development and for coping with external abiotic stresses [6]. MYB transcription factors are the largest class of transcriptional regulators in plants and play important roles in growth, metabolism, and resistance to stress. MYB transcription factors have one or more conserved structural domains consisting of 51 or 52 amino acid residues with a helix-helix-turned-helical spatial pattern. The MYB gene family is divided into four subfamilies: 1R-MYB (MYB-related), 2R-MYB (R2R3-MYB), 3R-MYB (R1R2R3-MYB), and 4R-MYB (R1R2R2R1/2-MYB). 2R-MYB transcription factors are the most abundant type of MYB transcription factors in plants $[7,8]$.

Many MYB gene families have been identified in various plants and analyzed genome-wide. As of December 2020, 69,027 MYB genes were officially registered in the NCBI database (https://www.ncbi.nlm.nih.gov/). Physiological, biochemical, and molecular tests indicated that many MYB transcription factors are associated with plant growth, stress resistance, and the synthesis of secondary metabolites [9]. For example, AtMYB2 [10] and AtMYB68 [11] improved drought tolerance and boric acid tolerance in Arabidopsis; OSARM1 [12] improved resistance to arsenic stress in rice; AtMYB58 [13], AtMYB123 [14], and AtMYB11 [15] are associated with the Arabidopsis phenylpropanoid metabolic pathway, proanthocyanidin synthesis, and flavonol biosynthesis, respectively. While the MYB gene family has been intensively studied and explored in various plants, no systematic studies have yet been reported for many hemp crops, particularly industrial hemp.

Hemp (Cannabis sativa L.) is a variety of the genus Cannabis in the family Cannabaceae and is widely used in textiles, medicines, skin care, and health care [16]. A tetrahydrocannabinol (THC) content of less than $0.3 \%$ in hemp indicates an industrial hemp and is considered to be of no use 
as a drug hemp variety [17]. Internationally, industrial hemp has been grown in soil contaminated with $\mathrm{Cd}$ to remediate the soil, producing economic benefits while also preventing the heavy metal from entering the human food chain [18]. The MYB transcription factor is the most important transcription factor for plant development and stress resistance. Systematic identification of the whole hemp genome and related research into Cd stress is necessary. In recent years, studies have found that exogenous heavy metal stress can increase CBD, a representative cannabinoid, content in hemp [19]. One of the objectives of this study was to determine whether Cd stress leads to changes in the content of cannabinoids by affecting the expression of MYB genes.

The aim of this study was to conduct a genome-wide analysis of hemp MYB genes using bioinformatics and to analyze their genetic structural differences and evolutionary relationships, and conjoint analysis with Cd-stress and cannabinoid synthesis. In this study, 115 MYB genes were identified from the hemp genome by analyzed their evolutionary relationships and gene structure. Their evolutionary relationships with dicotyledonous soybean (Glycine max), Raymond's cotton (Gossypium raimondii) and monocotyledonous maize (Zea mays L.), and rice (Oryza sativa L.) were compared. simultaneously, we analyzed transcriptome data of two hemp varieties with Cd-resistance, we identified 7 CsMYB genes that may potentially play a vital role in Cd-stress resistance. More importantly, by determining the cannabinoid changes of hemp flowers and leaves after $\mathrm{Cd}$ treatmet, expression patterns of CsMYB genes of hemp varieties with different cannabidiol contents, measured the expression levels of CsMYB genes in, roots, stems, leaves, flowers. we identified a $C S M Y B$ gene that may regulate cannabinoid biosynthesis. This study can provide a meaningful reference for potato breeding improvement.

\section{Materials and Methods}

\section{Identification of hemp MYB genes}

The hemp genome [20] and genome annotation (assembly number: GCA_900626175.2) were downloaded from the NCBI database (https://www.ncbi.nlm.nih.gov/). The MYB gene sequences of rice and soybean were downloaded from the PlantTFDB [21] database (http://planttfdb.gao-lab.org/) and compared to the hemp genome using the software TBtools [22], for blast sequences (E-value $<1 \mathrm{E}^{-5}$ ), to screen the candidate genes of MYB family in hemp. The 
candidate genes were submitted to the UniProt database (https://www.uniprot.org/) for batch comparison to verify whether the candidate genes contained MYB-conserved structural domains and to remove false-positive genes. Physicochemical properties were analyzed using the online software ExPASy Proteomics (http://web.expasy.org/compute_pi).

\section{Construction of phylogenetic tree and gene structure analysis of hemp MYB gene}

Hemp MYB protein sequences were imported into the software MEGA7.0 [23], and the results of the comparison were used to construct a phylogenetic tree using the neighbor-joining (NJ) method (execution parameter: bootstrap method 1000) [24,25]. The software FigTree [26] was used to refine the embellished evolutionary tree. Protein structure prediction was performed using the online software NCBI-CDD (https://www.ncbi.nlm.nih.gov/cdd/) to detect the protein structural domain of hemp MYB (E-value < 0.01). The conserved motifs of the hemp MYB gene were analyzed using the online software MEME (http://meme-suite.org) with the prediction number set to 10 . The software TBtools was used to extract the relevant coding sequences (CDS) and untranslated regions (UTRs) of hemp MYB from the hemp genome annotation files. TBtools was then used to combine the evolutionary tree, protein structural domain, gene conserved motifs, CDS, and UTR to construct an overall comparison map of MYB evolutionary relationships and structures.

\section{Chromosomal distribution and synteny analysis of hemp MYB gene}

TBtools software was used to extract MYB gene location information from the hemp genome files and gene annotation files and to construct a physical map of the MYB gene on the chromosomes. TBtools, MCScanX [27] and Circos [28] were used to calculate and map the presence of tandem repeats of hemp MYB on chromosomes, presence of covariance genes between different chromosomes of hemp, and relationship between hemp and soybean, Raymond's cotton, maize, and rice.

\section{Analysis of MYB gene expression in Yunnan hemp no. 1 and Inner Mongolia small grain hemp under Cd stress}

The transcriptomic data of Yunnan hemp no. 1 (Cd-tolerant) and Inner Mongolia small grain 
hemp (Cd-sensitive) were selected under normal environment and Cd-stress [18]. The raw sequencing data were converted into Sequenced Reads or Raw Reads using CASAVA base calling analysis and then filtered again to obtain high-quality, pure reads. The quality-controlled data were compared to the Hemp (version GCF_900626175.2) reference genome [20] using both TopHat2 (http://ccb.jhu.edu/software/tophat/index.shtml) and HISAT2 (http://ccb.jhu.edu/software/hisat2/index.shtml) software, and mapped reads were spliced using String Tie [29] or Cufflinks [30]. The raw transcriptome data were matched to the hemp MYB genes obtained from genome-wide analysis, and the number of reads was counted and further converted to FPKM values [31] for comparative analysis. The expression of homologous genes, which displayed consistent change trends under Cd stress, was compared between the two hemp varieties, and the gene-to-gene correlation coefficients were calculated using the Spearman correlation algorithm [32,33] to infer the key genes responsible for Cd tolerance, based on the degree of association between the genes.

\section{Changes in the CBD and CBDA contents of hemp flowers and leaves after Cd treatment}

Adult hemp plants (Yunnan hemp no. 1 grow with greenhouse), grown to the flowering stage, were transferred to soil containing $\mathrm{Cd}$ at a concentration of $100 \mathrm{mg} / \mathrm{kg}$, and the $\mathrm{CBD}$ and the $\mathrm{CBD}$ precursor (CBDA) Percentage contents in the flowers and leaves were measured after 15 days of treatment [34].

\section{MYB gene expression analysis of hemp varieties with different cannabidiol contents}

Two hemp varieties with different CBD contents were selected: H8 (higher CBD content) and FM (lower CBD content), and the expression of 14 MYB genes was examined in randomized samples from both the hemp flowers and leaves. The primers were designed using Primer 3 plus (Table S5), with DHS2 [35] as the internal reference gene, and the upstream and downstream primer sequences were sense: 5-TTGTCAACCAACCTCGTGTC-3; antisense: 5-GAGGGCTCTCTCGTAGCACATC-3. The tests were conducted using a BIO-RAD CFX Connect fluorescence qPCR instrument. The period taken was the flowering period of the two hemp species, and RNA was extracted using the Aidlab kit. cDNA was synthesized using the 
Takara reverse transcription kit, and real-time fluorescence quantitative PCR was verified using the Takara kit. The reaction conditions were as follows: pre-denaturation at $95{ }^{\circ} \mathrm{C}$ for $2 \mathrm{~min}$, denaturation at $95{ }^{\circ} \mathrm{C}$ for $15 \mathrm{~s}$, annealing at $60{ }^{\circ} \mathrm{C}$ for $30 \mathrm{~s}$, and 40 cycles. The relative expression of the target genes was calculated using the $2^{-\Delta \Delta} \mathrm{Ct}$ method.

\section{Tissue-specific expression analysis of key MYB genes}

The key MYB genes selected in 4.4 and 4.6 were subjected to tissue-specific expression analysis in Yunnan hemp no. 1 grow with greenhouse. The methods previously described in 4.6 were used to determine their expression in tissues collected from the roots, stems, leaves, and flowers (Table S5).

\section{Results}

\section{Identification of hemp MYB genes}

Using gene sequence comparison and conserved structural domain analysis, 115 MYB genes were identified from hemp and named CsMYB001-CsMYB115 based on the information obtained from the CDS fragments (Table s1). The isoelectric point of the hemp MYB proteins ranged from 4.66 to 10.06 , with an average protein isoelectric point of 7.13 ; the protein length ranged from 78 to 1258 aa, with an average length of 382.50 aa; and the molecular mass ranged from $8,907.78$ to $141,540.30 \mathrm{kDa}$, with an average molecular mass of 43,076.59 kDa (Table S1).

\section{Phylogenetic tree construction and structure analysis of the MYB gene family}

To understand the evolutionary relationships among hemp MYB genes, an evolutionary tree was constructed with the 115 hemp MYB genes, which were divided into three subfamilies; 1R-MYB/MYB-related, 2R-MYB/R2R3-MYB, and 3R-MYB (Figure 1).

The 2R-MYB subfamily had the most members, consisting of 88 genes, the 3R-MYB subfamily had the least members with 8 genes, and the 1R-MYB subfamily contained 17 genes. CsMYB097 and CsMYB098 were identified but were not placed into any of the three subfamilies because of the dispersion of the conserved structural domains of the genes; however, CsMYB097 was similar to the combination of two 2R-MYB genes, and CsMYB098 resembled the R2R3-MYB structural domain divided into two segments. Interestingly, CsMYB024, CsMYB037, CsMYB064, 
and $C s M Y B 112$ were 3R-MYB genes that appeared in the 2R-MYB subfamily in the evolutionary tree, and CsMYB023 and CsMYB094 were 1R-MYB genes that also appeared in the 2R-MYB subfamily in the evolutionary tree, presumably evolving from the more closely related 2R-MYB subfamily. Figure 2 illustrates that the gene structures within each subfamily are basically the same, and the conserved motifs are also similar, indicating that the genes within a subfamily have similar functions. Among the hemp MYB genes, $6.96 \%(8 / 115)$ of the genes had no introns and, presumably, had similar functions and expression patterns. While motifs 3,4 , and 2 of the hemp MYB gene members are R2 conserved structural domains, motif 1 is an R3 conserved structural domain (Figure 3).

\section{Chromosomal distribution and synteny analysis of hemp MYB genes}

Analysis of MYB gene locations on hemp chromosomes showed that the MYB genes were distributed on 10 chromosomes, with no significant correlation to chromosome length (Figure 4), and loci were primarily at the ends of the chromosomes. Chromosome NC2 contained the most MYB genes with 18, NC9 had the least MYB genes with only 3, and the other chromosomes comprised 6 to 17 MYB genes. A small number of tandem duplications were found on chromosomes NC1, NC2, and NC10, which accounted for 5.22\% of all MYB genes. Analysis of covariance between hemp chromosomes revealed 21 pairs of covariates (Figure 5). With the exception of CsMYB017, CsMYB074, CsMYB078 and CsMYB095, which were interrelated to the 1R-MYB and 2R-MYB subfamilies, the remaining 19 pairs of covariates belonged to the $2 \mathrm{R}$ subfamily, indicating that the 2R-MYB family genes have undergone several gene duplication events leading to the expansion of MYB gene family members. No codominant genes were found in the 3R-MYB subfamily with other subfamilies. The prediction of paralogous genes revealed that CsMYB113, CsMYB095, CsMYB072, and CsMYB060 were covalently related to several genes, and all belonged to the 2R-MYB subfamily, which may be the more primitive genes in the MYB family and have made important contributions to the expansion of the 2R-MYB subfamily. To further understand the evolutionary mechanism of the MYB family in hemp, covariance maps of the MYB family were constructed for hemp and Raymond's cotton, soybean, rice, and maize

(Figure 6). The covariance results indicated that hemp had direct homologous genes with each of the plants as follows: 84 pairs with Raymond's cotton, 137 pairs with soybean, 12 pairs with rice, 
and 7 pairs with maize, which is also consistent with the evolutionary relationship between hemp and the four corresponding plants (Table S2). Synteny analysis of the five plants revealed 60 MYB genes that were unique to hemp and may have been derived from tandem repeat amplification of the original MYB genes of hemp, which may be relevant for understanding the functional differences between the MYB genes of hemp and other plants [36]. Conversely, CsMYB005, CsMYB013, CsMYB016, CsMYB041, CsMYB053, and CsMYB081 were identified in all five plants, suggesting that these genes may be MYB genes that existed before the divergence of dicotyledons from monocotyledons [37]. In contrast, the CSMYB113, which has a covariance between hemp chromosomes, was present in all three species except in maize, where no covariance was found. None of the remaining paralogous hemp genes were present in the analysis of covariance with the other four species, strongly indicating that CsMYB113 may have arisen by amplification.

\section{Expression analysis of MYB genes in two hemp varieties under Cd stress}

In order to understand the expression of hemp MYB and CBD synthesis genes under normal and Cd stress conditions, transcriptome determination was performed on plants of Yunnan hemp no. 1 and Inner Mongolia small grain hemp under the two environments. Genes with zero expression in each group were deleted, resulting in 107 MYB genes obtained for Yunnan hemp no. 1 and 111 MYB genes obtained for Inner Mongolia small grain hemp (Table S3, Table S4). The expression amounts reported for all of the genes in Table S3 and Table S4 are the average of three replicates. The transcriptome data results for Yunnan hemp no. 1 (Table S3) showed a decrease $(39.25 \%)$ in the expression of 42 genes and a decrease in ploidy ranging from 0.03 to 0.95 , among which the expression of 16 genes changed significantly $(\mathrm{p}<0.05)$; 65 genes were upregulated $(60.75 \%)$ with the upregulation ploidy ranging from 1.07 to 1396.11 , among which the expression of 43 genes changed significantly $(\mathrm{p}<0.05)$. The transcriptome data for Inner Mongolia small grain hemp (Table S4) indicated the expression of 48 genes was decreased (37.84 \%) with ploidy ranging from 0.06 to 0.99 , among which 13 genes were significantly differentially expressed; the expression of 63 genes was upregulated (62.16\%) with ploidy ranging from 1.01 to 56.34 , among which 21 genes were significantly differentially expressed. These changes indicate that external 
stress had a significant impact on the expression of MYB genes with the MYB genes of the Cd-tolerant varieties undergoing more significant change than those of the Cd-sensitive varieties, further indicating that MYB genes play an important role in the Cd tolerance of hemp.

\section{Identification of key Cd-tolerance MYB genes and correlation analysis}

Based on the expression trends of MYB genes in two varieties of hemp under different environmental conditions (part 2.4), the immediate homologous genes in two hemp varieties with consistent change trends in expression were selected for comparison. As shown in Figure 7a, 48 genes were upregulated in two varieties, and Figure $7 \mathrm{~b}$ shows that these 48 genes were upregulated more in cloud hemp, from the top to the bottom of the CsMYB052-CsMYB006 and CsMYB062-CsMYB053 genes. As shown in Figure 8a, 28 genes were downregulated in two varieties, and Figure $8 \mathrm{~b}$ shows that these 28 genes were downregulated more in Yunnan hemp No.1, from the top to the bottom of the CsMYB025-CsMYB023 and CsMYB010-CsMYB024 genes. These hemp MYB genes that displayed greater variation in expression may be the key MYB genes that code for greater $\mathrm{Cd}$ tolerance. The key differential genes identified were subjected to Spearman correlation analysis, and based on the correlation between genes, it was hypothesized that CsMYB045, CsMYB016, CsMYB067, and CsMYB098 may be the key genes in the upregulated MYB network (Figure 7c), whereas CsMYB010, CsMYB061, and CsMYB005 may be the key genes in the downregulated MYB network (Figure 8c).

\section{Changes in the contents of CBD and CBDA after Cd treatment in hemp}

CBD is a unique secondary metabolite in hemp that can be used to treat a variety of diseases. It is currently an important plant secondary metabolite of international concern. Three asexual hemp plants grown to the flowering stage were transferred into soil containing $\mathrm{Cd}$ at a concentration of $100 \mathrm{mg} / \mathrm{kg}$, and three asexual hemp plants were grown in a normal environment. Fifteen days later, the CBD and cannabidolic acid (CDBA) contents in the flowers and leaves of the six asexual hemp plants were measured. As seen in Figure 9, the CBD and CDBA contents in flowers increased in the Cd-stressed environment, whereas the CBD and CDBA contents in leaves decreased in the Cd-stressed environment. The change in CBD content of the leaves was 
significant $(\mathrm{p} \leqslant 0.05)$, which indicates that external Cd stress may affect the CBD content of hemp, even though the changes in contents of the other cannabinoids were not statistically significant. This may have been because the tolerance to $\mathrm{Cd}$ in adult hemp plants was high and the external $\mathrm{Cd}$ stress treatment time was too short.

\section{Analysis of MYB gene expression in hemp varieties with high and low cannabidiol contents}

The application of external Cd-stress to hemp plants was found to alter the plants' CBD and CBDA contents and the expression of a large number of hemp MYB genes; thus, MYB genes might be associated with the production of plant secondary metabolites. To investigate MYB genes that might be associated with CBD production, we randomly selected 14 MYB genes: CsMYв009, CsMYв017, CsMYB024, CsMYB028, CsMYB032, CsMYB033, CsMYB035, CsMYB037, CsMYB046, CsMYB047, CsMYB050, CsMYB053, CsMYB062, and CsMYB067 and determined their expression in the flowers and leaves of hemp varieties (Figure 11) with high CBD content (H8) versus low CBD content (FM) (Figure 10). The expression of CsMYB063 and CsMYB067 was detected in both the H8 and FM hemp varieties.

The CBD content of both the flowers and the leaves in $\mathrm{H} 8$ was higher than that in FM; thus, the MYB genes with the same trend of variation in the flowers and leaves of both hemp cultivars selected for analysis were found to be strongly correlated with CBD content. Measuring gene expression found that the trends in expression of CsMYB024, CsMYB028, and CsMYB062 in the flowers and leaves were consistent among the 14 genes, and all the three genes in cloud hemp showed significant differential expression under Cd stress, indicating that $\mathrm{Cd}$ stress may affect the expression of MYB genes which leads to changes in CBD synthesis. The expression of these three genes did not change significantly in the Inner Mongolia small grain hemp under $\mathrm{Cd}$ stress, indicating that the change in CBD content may be related to the Cd-tolerance of hemp.

\section{Comparison of the expression of the key Cd-tolerant MYB genes of hemp in different parts of the plant}

To further clarify the expression of key Cd-tolerant MYB genes in different parts of hemp, the seven MYB genes selected in subsection 2.5 and the three MYB genes selected in subsection 
2.7 were used to determine the relative expression of the key MYB genes in the roots, stems, leaves, and flowers during the flowering period of hemp (Figure 12). The clustering of similar gene expression patterns indicated that the key MYB genes that were upregulated or downregulated had similar expression patterns. The four upregulated key MYB genes all had higher expression in the roots, whereas the three downregulated key MYB genes were all in the stem and had high expression in the flowers. Among them, CsMYB024 had a similar expression pattern to the downregulated key MYB genes, and CSMYB028 had a similar expression pattern to the upregulated key MYB genes.

\section{Discussion}

\section{Identification and evolutionary analysis of the CsMYB gene family}

MYB transcription factors are one of the largest transcription factor families in the plant kingdom and influence the regulation of plant growth, development, and response to different stresses [38-40]. With the rapid development of sequencing technology, increasing plant genomes have been sequenced, and the MYB transcription factor gene members were identified using genome-wide analysis in plants such as Arabidopsis thaliana (197 members) [41], Solanum tuberosum L. (158 members) [42], Oryza sativa L. (155 members) [41], soybean (244 members) [43], Physcomitrella patens (116 members) [44], and Brassica napus L. (249 members) [45,46]. In this study, 115 members of the MYB gene family were identified in hemp with an $876.14 \mathrm{Mb}$ genome size. Considering the different genome sizes and numbers of MYB genes in these plant species, we hypothesized that the number of MYB genes is not entirely associated with their genome.

Gene duplication events, which result from unequal crossing-over, duplication-dependent chromosome breakage, and nonhomologous exchange, are the main factors contributing to the expansion of gene families [47]. In a previous study, a large number of tandem-duplicated gene pairs and segmental duplications were identified in some plants, including potato, pear, apple, grape, physic nut, and Gossypium raimondii [48,49]. Similarly, 3 pairs of tandem-duplicated genes and 21 segmental-duplicated genes were identified in the hemp genome (Figure 4, Figure 5). Taken together, we speculate that segmental duplication was the main factor during the 
evolutionary process that resulted in the expansion of the MYB family in hemp.

\section{Potential functions analysis of CsMYB in Cd-Stress response and regulate cannabinoid}

\section{biosynthesis}

To further explore the evolutionary pattern of MYB genes in hemp, synteny analyses were performed between the MYB genes of hemp and four representative plants. CsMYB genes were found to have more homologous gene pairs with MYB genes in Gossypium raimondii and Glycine max than those in Oryza sativa L. and Zea mays L. (Figure 6). One possible reason for these results is that hemp, Gossypium raimondii, and Glycine max are dicot plants, whereas Oryza sativa L. and Zea mays L. are monocot plants, and the results are similar to the synteny analyses results for potato, poplar, and wheat [50,51]. Among these homologous genes, CsMYB005, CsMYB013, CsMYB016, CsMYB041, CsMYB053, and CsMYB081 were identified in all five plants, which suggests that these genes existed before the divergence of dicot and monocot plants, and some MYB genes found in different plants may have originated from a common ancestor.

Hemp can be used for the remediation of heavy metal-contaminated soils [52], and MYB genes have been widely reported to be involved in the regulation of plant defense responses to heavy metal stress [53]. In our study, the expression of most CsMYB genes was altered in both Cd-sensitive and Cd-tolerant hemp varieties in response to Cd-stress. Although the trends in expression patterns were similar in Cd-tolerant and Cd-sensitive hemp varieties, the induction of transcript levels was more pronounced in Cd-tolerant varieties than in Cd-sensitive varieties. Based on correlation coefficient analysis, seven $C S M Y B$ genes were further hypothesized to be the key regulators in response to Cd stress (Figure 7, Figure 8). Four CsMYB genes were upregulated under Cd stress, with CsMYB016 having a direct homolog in five species, CsMYB067 having a direct homolog in three species, CsMYB098 having direct homologs in two species, and CsMYB045 having no direct homologs. These results indicated that the four genes were in turn less primitive. Both CSMYB016 and CSMYB067 were involved in one amplification of paralogous homologs within hemp species, suggesting they may have evolved along with new functions in hemp, while CSMYB067 displayed similarity to genes in Arabidopsis that may be related to cytokinin [36] or abscisic acid [37] and are significantly upregulated under salt stress [38], thus 
providing additional evidence that these four genes may be the key genes in regulating $\mathrm{Cd}$ tolerance. Three other CsMYB genes were downregulated under Cd stress, with CsMYB005 having direct homologs in five species, CsMYB061 having direct homologs in three species, and CsMYB010 having no direct homologs, indicating that the original degree of the three genes was reduced in order. Similar genes in Arabidopsis are associated with cell growth and cell wall development [54,55]; however, the functions of these genes in hemp need further validation.

Cannabinol is a secondary metabolite and one of the main phenolic compounds in hemp. In recent years, CBD sales has captured an increased segment of the market, and scientific research into the use of CBD continues to expand. External stress can affect the content of plant secondary metabolites: when the industrial hemp is exposed to Cd stress, there are significant changes in cannabinol content and the expression of MYB genes, which are the key transcription factors for the synthesis of secondary metabolites. Although the pathway of cannabinol synthesis has been clarified, the related genes (such as MYB genes) that regulate its content within the plant are not yet clear. We found that the expression of CsMYB024, CsMYB028, and CsMYB062, selected from 2.7, was higher in both the leaves and flowers of hemp with high CBD content than in those with low CBD content. In the examination of tissue-specific expression, CsMYB024 was found to be relatively highly expressed in the flowers and leaves, both of which are organs with high CBD accumulation, indicating that this gene may be associated with CBD synthesis. While the expression of CsMYB024 showed a decreasing trend in the Cd-stressed hemp of both varieties, the $\mathrm{CBD}$ and CBDA contents in the hemp leaves were significantly downregulated under Cd stress. It is speculated that CsMYBO24 may be involved in the regulation of a Cd-mediated decrease in CBDA content, but its executive function is not yet clear and needs to be verified in future experiments.

A total of 115 CsMYB genes were identified from the hemp genome and were further studied using bioinformatics and tissue-specific expression analysis. Furthermore, the potential function of $C S M Y B$ genes in response to $\mathrm{Cd}$ stress and the regulation of CBDA biosynthesis were explored. Several potential candidate genes for participating in $\mathrm{Cd}$ stress responses or regulating the synthesis of CBDA have been identified and characterized. Our research provides insight into the functional analysis of CsMYB genes in hemp and indicates areas in which further analysis is 
needed. The next step of this study can increase the concentration gradient and time gradient of hemp Cd-stress, and it is also necessary to carry out transgenic verification of certain genes.

\title{
Supplementary information
}

Table S1. List of the 115 CsMYB genes identified in this study.

Table S2. One-to-one orthologous relationships between hemp and Raymond's cotton.

Table S3. MYB gene expression in Yunnan hemp No.1.

Table S4. MYB gene expression in Inner Mongolia small hemp.

Table S5. Primers of sequences.

\begin{abstract}
Abbreviations
MYB: v-myb avian myeloblastosis viral; Cd: Cadmium; cDNA: Complementary DNA; MW: Molecular weight; CBD: cannabidiol; CBDA: cannabidiol acid; FPKM: Fragments per kilobase of transcript per million mapped reads; CDS: coding sequences; UTRs: untranslated regions.
\end{abstract}

\section{Acknowledgements}

Not applicable.

\section{Authors' contributions}

M.Y., G.P., S.H. and D.L. conceived the original research plan, J.T., L.Z., Z.L. and H.J. performed experiments, H.T., L.C., J.L. and A.C. carried out the data analysis; M.Y. and G.P. wrote the article, Y.D. and C.Z. revised the manuscript. All authors contributed to the research and read and approved the final manuscript.

\section{Funding}

This research was mainly funded by the China Agriculture Research System for Bast and Leaf Fiber Crops (CARS-16-E-02), National Natural Science Foundation of China (Grant No. 31871674), Central Public-interest Scientific Institution Basal Research (1610242020003).

\section{Availability of data and materials}

The RNA sequencing (RNA-seq) data of each CsMYB gene were obtained from previous studies in different hemp varieties (https://www.sciencedirect.com/science/article/pii/S092666901930648X).

\section{Ethics approval and consent to participate}

Not applicable.

\section{Consent for publication}

Not applicable.

\section{Competing interests}

The authors declare that they have no competing interests.

\section{Author details}


${ }^{1}$ Institute of Bast Fiber Crops, Chinese Academy of Agricultural Sciences, Changsha 410000, China

${ }^{2}$ Key Laboratory of Biological and Processing for Bast Fiber Crops, Ministry of Agriculture and Rural Affairs, Changsha 410000, China

\section{References}

1. Saijo Y, Loo EP. Plant immunity in signal integration between biotic and abiotic stress responses. New Phytol. 2020; 225: 87-104.

2. Meena RAA, Sathishkumar P, Ameen F, Yusoff ARM, Gu FL. Heavy metal pollution in immobile and mobile components of lentic ecosystems-a review. Environ Sci Pollut Res Int. 2018; 25: 4134-4148.

3. Liu N, Dong L, Deng X, Liu D, Liu Y, Li M, Hu Y, Yan Y. Genome-wide identification, molecular evolution, and expression analysis of auxin response factor (ARF) gene family in Brachypodium distachyon L. BMC Plant Biol. 2018; 18: 336.

4. Himuro Y, Ishiyama K, Mori F, Gond T, Takahashi F, Shinozaki K, Kobayashi M, Akashi R. Arabidopsis galactinol synthase AtGolS2 improves drought tolerance in the monocot model Brachypodium distachyon. J Plant Physiol. 2014; 171: 1127-31.

5. Paulose B, Kandasamy S, Dhankher OP. Expression profiling of Crambe abyssinica under arsenate stress identifies genes and gene networks involved in arsenic metabolism and detoxification. BMC Plant Biol. 2010; 10 : 108.

6. Wang LJ, Lu WX, Ran LY, Dou LW, Yao S, Hu J, Fan D, Li CF, Luo KM. R2R3-MYB transcription factor MYB6 promotes anthocyanin and proanthocyanidin biosynthesis but inhibits secondary cell wall formation in Populus tomentosa(Article).Plant Journal. 2019; 99: 733-751.

7. Dubos C, Stracke R, Grotewold E, Weisshaar B, Martin C, Lepiniec L. MYB transcription factors in Arabidopsis. Trends Plant Sci. 2010; 15: 573-81.

8. Liu J, Osbourn A, Ma P. MYB Transcription Factors as Regulators of Phenylpropanoid Metabolism in Plants. Mol Plant. 2015, 8: 689-708.

9. Guo H, Wang Y, Wang L, Hu P, Wang Y, Jia Y, Zhang C, Zhan Y, Zhang Y, Wang C, Yang C. Expression of the MYB transcription factor gene BplMYB46 affects abiotic stress tolerance and secondary cell wall deposition in Betula platyphylla. Plant Biotechnol J. 2017; 15: 107-121.

10. Baek D, Chun HJ, Kang S, Shin G, Park SJ, Hong H, Kim C, Kim DH, Lee SY, Kim MC, Yun DJ. A Role for Arabidopsis miR399f in Salt, Drought, and ABA Signaling. Mol Cells. 2016; 39: 111-8.

11. Nozawa A, Miwa K, Kobayashi M, Fujiwara T. Isolation of Arabidopsis thaliana cDNAs that confer yeast boric acid tolerance. Biosci Biotechnol Biochem. 2006; 70: 1724-30.

12. Wang FZ, Chen MX, Yu LJ, Xie LJ, Yuan LB, Qi H, Xiao M, Guo W, Chen Z, Yi K, Zhang J, Qiu R, Shu W, Xiao S, Chen QF. OsARM1, an R2R3 MYB Transcription Factor, Is Involved in Regulation of the Response to Arsenic Stress in Rice. Front Plant Sci. 2017; 8: 1868.

13. Segarra G, Van der Ent S, Trillas I, Pieterse CM. MYB72, a node of convergence in induced systemic resistance triggered by a fungal and a bacterial beneficial microbe. Plant Biol (Stuttg). 2009; 11: 90-6.

14. Paz-Ares J, Ghosal D, Wienand U, Peterson PA, Saedler H. The regulatory c1 locus of Zea mays encodes a protein with homology to myb proto-oncogene products and with structural similarities to transcriptional activators. EMBO J. 1987; 6: 3553-8.

15. Grotewold E, Athma P, Peterson T. Alternatively spliced products of the maize $\mathrm{P}$ gene encode proteins with homology to the DNA-binding domain of myb-like transcription factors. Proc Natl Acad Sci U S A. 1991; 88: 4587-91. 
16. Schluttenhofer C, Yuan L. Challenges towards Revitalizing Hemp: A Multifaceted Crop. Trends Plant Sci. 2017; 22: 917-929.

17. Sanchez L, Baltensperger D, Kurouski D. Raman-Based Differentiation of Hemp, Cannabidiol-Rich Hemp, and Cannabis. Anal Chem. 2020; 92: 7733-7737.

18. Huang YM, Li DF, Zhao LN, Chen AG, Li JJ, Tang HJ, Pan G, Chang L, Deng Y, Huang SQ. Comparative transcriptome combined with physiological analyses revealed key factors for differential cadmium tolerance in two contrasting hemp (Cannabis sativa L.) cultivars. Industrial Crops and Products. 2019; 104.

19. Husain R, Weeden H, Bogush D, Deguchi M, Soliman M, Potlakayala S, Katam R, Goldman S, Rudrabhatla S. Enhanced tolerance of industrial hemp (Cannabis sativa L.) plants on abandoned mine land soil leads to overexpression of cannabinoids. PLoS One. 2019; 14: e0221570.

20. Laverty KU, Stout JM, Sullivan MJ, Shah H, Gill N, Holbrook L, Deikus G, Sebra R, Hughes TR, Page JE, van Bakel H. A physical and genetic map of Cannabis sativaidentifies extensive rearrangements at the THC/CBD acid synthase loci. Genome Res. 2019; 29: 146-156.

21. Tian F, Yang DC, Meng YQ, Jin J, Gao G. PlantRegMap: charting functional regulatory maps in plants. Nucleic Acids Res. 2020; 48: D1104-D1113.

22. Chen C, Chen H, Zhang Y, Thomas HR, Frank MH, He Y, Xia R. TBtools: An Integrative Toolkit Developed for Interactive Analyses of Big Biological Data. Mol Plant. 2020; 13: 1194-1202.

23. Wu GQ, Li ZQ, Cao H, Wang JL. Genome-wide identification and expression analysis of the WRKY genes in sugar beet (Beta vulgaris L.) under alkaline stress. PeerJ. 2019; 7: e7817.

24. Wächter Dieter, Melzer Andreas. Proposal for a subdivision of the family Psathyrellaceae based on a taxon-rich phylogenetic analysis with iterative multigene guide tree. 2020; 19: 1151-1265.

25. Saitou N, Nei M. The neighbor-joining method: a new method for reconstructing phylogenetic trees. Mol Biol Evol. 1987; 4: 406-25.

26. Ronquist F, Teslenko M, van der Mark P, Ayres DL, Darling A, Höhna S, Larget B, Liu L, Suchard MA, Huelsenbeck JP. MrBayes 3.2: efficient Bayesian phylogenetic inference and model choice across a large model space. Syst Biol. 2012; 61: 539-42.

27. Li B, Fan R, Yang Q, Hu C, Sheng O, et al. Genome-Wide Identification and Characterization of the NAC Transcription Factor Family in Musa Acuminata and Expression Analysis during Fruit Ripening. Int J Mol Sci. 2020; $21: 634$.

28. Krzywinski M, Schein J, Birol I, Connors J, Gascoyne R, Horsman D, Jones SJ, Marra MA. Circos: an information aesthetic for comparative genomics. Genome Res. 2009; 19: 1639-45.

29. Kovaka S, Zimin AV, Pertea GM, Razaghi R, Salzberg SL. Pertea M Transcriptome assembly from long-read RNA-seq alignments with StringTie2, Genome Biology. 2019; 20: 278.

30. Trapnell C, Roberts A, Goff L, Pertea G, Kim D, Kelley DR, Pimentel H, Salzberg SL, Rinn JL, Pachter L. Differential gene and transcript expression analysis of RNA-seq experiments with TopHat and Cufflinks. Nat Protoc. 2012; 7: 562-78.

31. Yano R, Nonaka S, Ezura H. Melonet-DB, a Grand RNA-Seq Gene Expression Atlas in Melon (Cucumis melo L.). Plant Cell Physiol. 2018; 59: e4.

32. Pripp AH. Pearsons eller Spearmans korrelasjonskoeffisienter [Pearson's or Spearman's correlation coefficients]. Tidsskr Nor Laegeforen. 2018: 138.

33. Hazra A, Gogtay N. Biostatistics Series Module 6: Correlation and Linear Regression. Indian J Dermatol. 2016, 61, 593-601.

34. Mandrioli M, Tura M, Scotti S, Gallina Toschi T. Fast Detection of 10 Cannabinoids by RP-HPLC-UV Method in Cannabis sativa L. Molecules. 2019; 24:2113. 
35. Ritesh Kumar Raipuria, Vajinder Kumar, Kadur Narayan Guruprasad, et al. Arabidopsis thaliana DHS2 (AT4G33510) gene promoter is highly wound responsive and requires a part of the first exon sequences for its function. 2018; 27: 241-247.

36. Zhao Q, Li M, Jia Z, Liu F, Ma H, Huang Y, Song S. AtMYB44 Positively Regulates the Enhanced Elongation of Primary Roots Induced by N-3-Oxo-Hexanoyl-Homoserine Lactone in Arabidopsis thaliana. Mol Plant Microbe Interact. 2016; 29: 774-785.

37. Hieno A, Naznin HA, Hyakumachi M, Higuchi-Takeuchi M, Matsui M, Yamamoto YY. Possible Involvement of MYB44-Mediated Stomatal Regulation in Systemic Resistance Induced by Penicillium simplicissimum GP17-2 in Arabidopsis. Microbes Environ. 2016; 31: 154-9.

38. Liu C, Xie T, Chen C, Luan A, Long J, Li C, Ding Y, He Y. Genome-wide organization and expression profiling of the R2R3-MYB transcription factor family in pineapple (Ananas comosus). BMC Genomics. 2017; 18 : 503.

39. Sun W, Ma Z, Chen H, Liu M. MYB Gene Family in Potato (Solanum tuberosum L.): Genome-Wide Identification of Hormone-Responsive Reveals Their Potential Functions in Growth and Development. Int J Mol Sci. 2019; 20: 4847.

40. Baillo EH, Kimotho RN, Zhang Z, Xu P. Transcription Factors Associated with Abiotic and Biotic Stress Tolerance and Their Potential for Crops Improvement. Genes (Basel). 2019; 10: 771.

41. Katiyar A, Smita S, Lenka SK, Rajwanshi R, Chinnusamy V, Bansal KC. Genome-wide classification and expression analysis of MYB transcription factor families in rice and Arabidopsis. BMC Genomics. 2012; 13: 544.

42. Yuhui Liua, Yuting Zengb, Yuanming Lic, Zhen Liua, Kui Lin-Wangd, Richard V. Espleyd; Andrew C. Alland, Junlian Zhangac. Genomic survey and gene expression analysis of the MYB-related transcription factor superfamily in potato (Solanum tuberosum L.). International Journal of Biological Macromolecules.2020; 164: 2450-2464.

43. Du H, Yang SS, Liang Z, Feng BR, Liu L, Huang YB, Tang YX. Genome-wide analysis of the MYB transcription factor superfamily in soybean. BMC Plant Biol. 2012; 12: 106-128.

44. Pu X, Yang L, Liu L, Dong X, Chen S, Chen Z, Liu G, Jia Y, Yuan W, Liu L. Genome-Wide Analysis of the MYB Transcription Factor Superfamily in Physcomitrella patens. Int J Mol Sci. 2020; 21: 975.

45. Boulos C, France D, Shengyi L, Parkin IAP, Haibao T, Xiyin W, Julien C, Harry B, Chaobo T, Birgit S. Plant genetics. Early allopolyploid evolution in the post-Neolithic Brassica napus oilseed genome. Science. 2014; 345 : 950-953.

46. Hajiebrahimi A, Owji H, Hemmati S. Genome-wide identification, functional prediction, and evolutionary analysis of the R2R3-MYB superfamily in Brassica napus. Genome. 2017; 60: 797-814.

47. Sun W, Ma Z, Chen H, Liu M. MYB Gene Family in Potato (Solanum tuberosum L.): Genome-Wide Identification of Hormone-Responsive Reveals Their Potential Functions in Growth and Development. Int J Mol Sci. 2019; 20: 4847.

48. Zhou C, Chen Y, Wu Z, Lu W, Han J, Wu P, Chen Y, Li M, Jiang H, Wu G. Genome-wide analysis of the MYB gene family in physic nut (Jatropha curcas L.). Gene. 2015; 572: 63-71.

49. He Q, Jones DC, Li W, Xie F, Ma J, Sun R, Wang Q, Zhu S, Zhang B. Genome-Wide Identification of R2R3-MYB Genes and Expression Analyses During Abiotic Stress in Gossypium raimondii. Sci Rep. 2016; 6: 22980.

50. Zhao K, Cheng Z, Guo Q, Yao W, Liu H, Zhou B, Jiang T. Characterization of the Poplar R2R3-MYB Gene Family and Over-Expression of PsnMYB108 Confers Salt Tolerance in Transgenic Tobacco. Front Plant Sci. 2020; 11: 571881. 
51. Wei Q, Chen R, Wei X, Liu Y, Zhao S, Yin X, Xie T. Genome-wide identification of R2R3-MYB family in wheat and functional characteristics of the abiotic stress responsive gene TaMYB344. BMC Genomics. 2020; 21: 792.

52. Khan AG. Promises and potential of in situnano-phytoremediation strategy to mycorrhizo-remediate heavy metal contaminated soils using non-food bioenergy crops (Vetiver zizinoides and Cannabis sativa). Int J Phytoremediation. 2020; 22: 900-915.

53. Xu Z, Dong M, Peng X, Ku W, Zhao Y, Yang G. New insight into the molecular basis of cadmium stress responses of wild paper mulberry plant by transcriptome analysis. Ecotoxicol Environ Saf. 2019; 171: 301-312.

54. Zhang W, Wang N, Yang J, Guo H, Liu Z, Zheng X, Li S, Xiang F. The salt-induced transcription factor GmMYB84 confers salinity tolerance in soybean. Plant Sci. 2020; 291: 110326.

55. Machemer K, Shaiman O, Salts Y, Shabtai S, Sobolev I, Belausov E, Grotewold E, Barg R. Interplay of MYB factors in differential cell expansion, and consequences for tomato fruit development. Plant J. 2011; 68: 337-50.

56. Zhong R, Ye ZH. MYB46 and MYB83 bind to the SMRE sites and directly activate a suite of transcription factors and secondary wall biosynthetic genes. Plant Cell Physiol. 2012; 53: 368-80. 


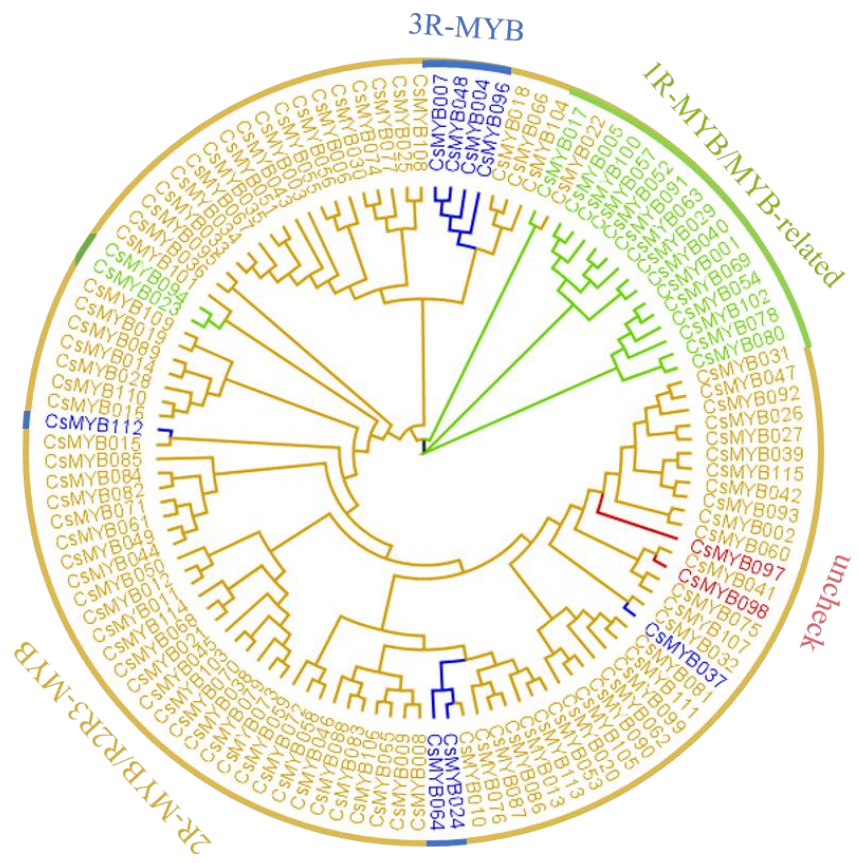

Figure 1. Phylogenetic tree of MYB family in hemp. Different colors indicate different subfamily, and different genes under the same color belong to the same subfamily. 

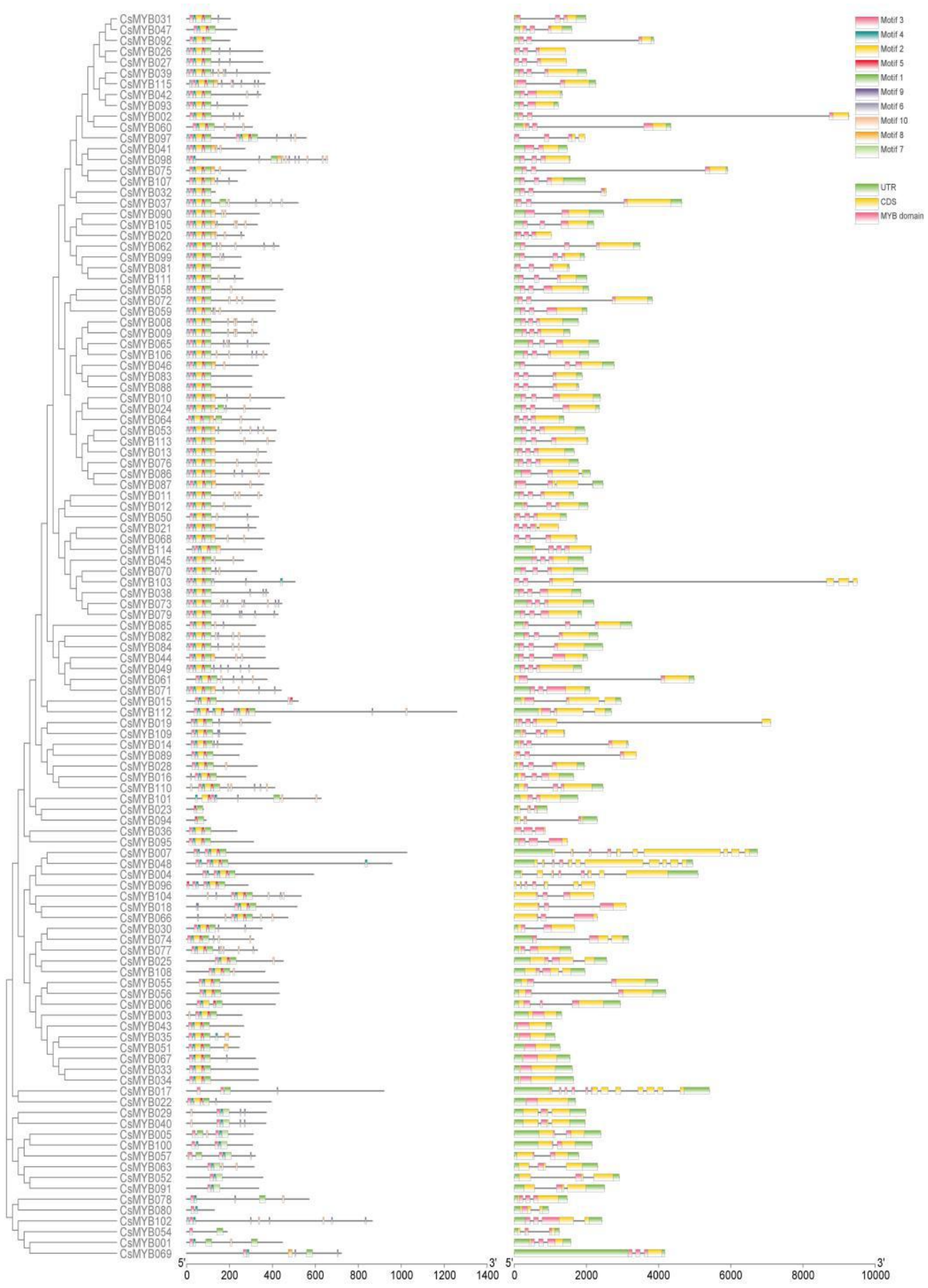

Figure 2. Gene structure analysis of MYB in hemp. Left for the evolutionary tree, based on the full-length sequences of hemp MYB proteins. Middle for gene structure analysis with motifs. Right for gene structure analysis with UTR, CDS, MYB domain. 


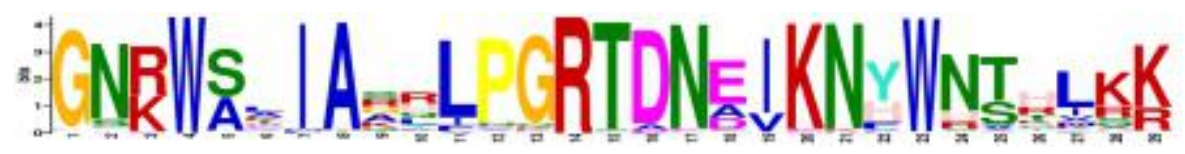

(a)

ACL RCKSCRLRW NYLR RIKRG FT:

(b)

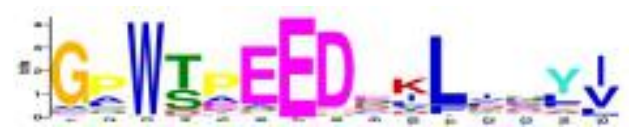

(c)

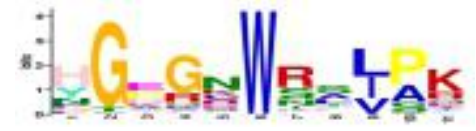

(d)

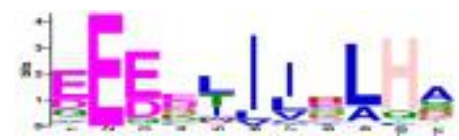

(e)

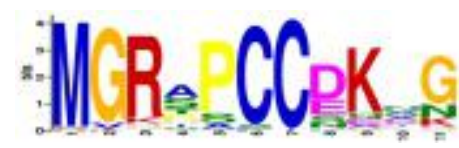

(f)

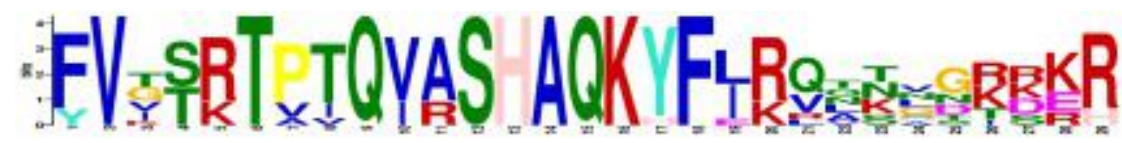

(g)

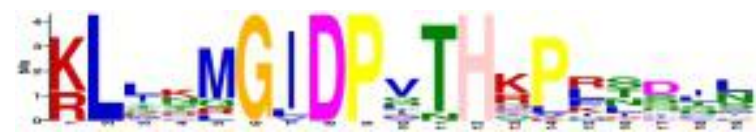

(h)

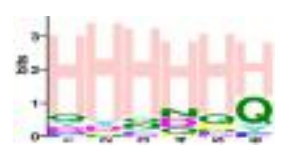

(i)

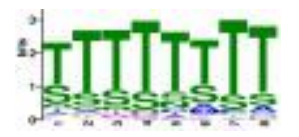

(j)

Figure 3. Gene motifs of CsMYB in hemp. From top to bottom, a to j means motif 1 to 10. 


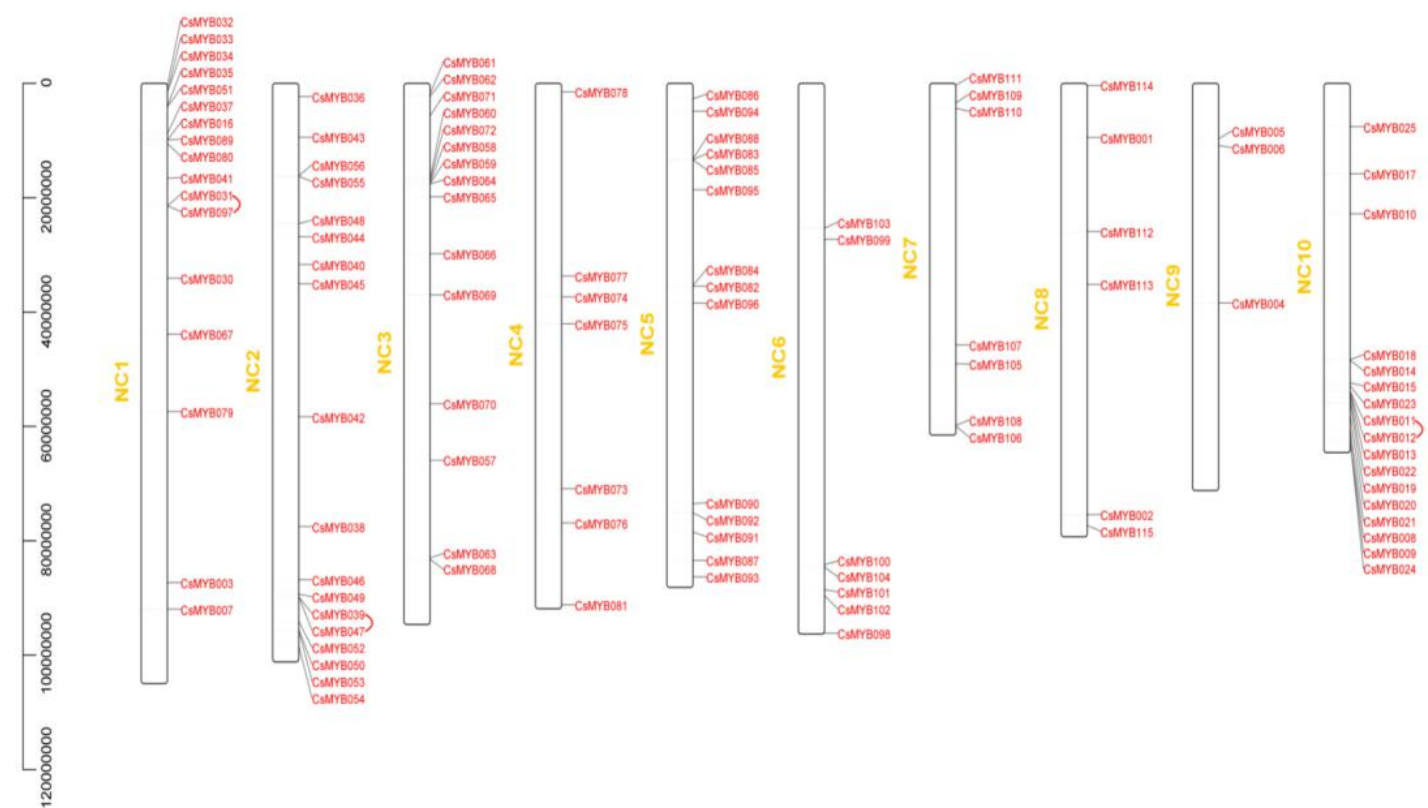

Figure 4. Chromosomal locations of MYB in hemp. genes on the same chromosome are connected by red lines, indicating that the related genes are tandem duplications. 


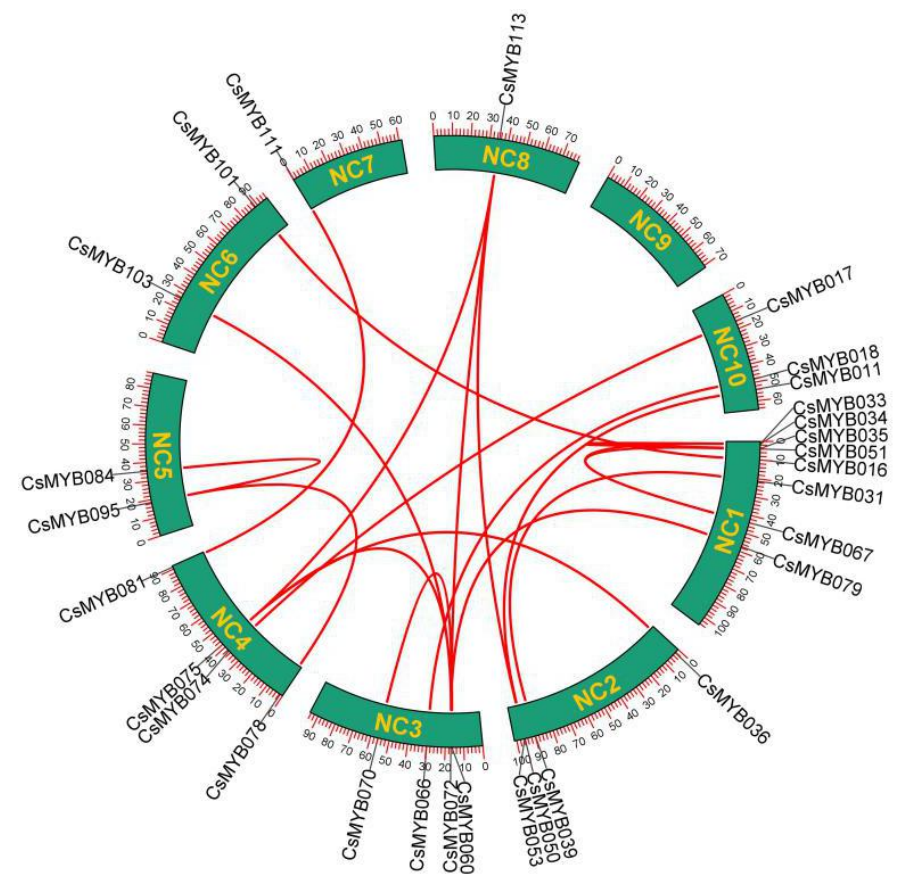

Figure 5. MYB synteny analysis among chromosomes in hemp, genes with red lines connected are covariates. 

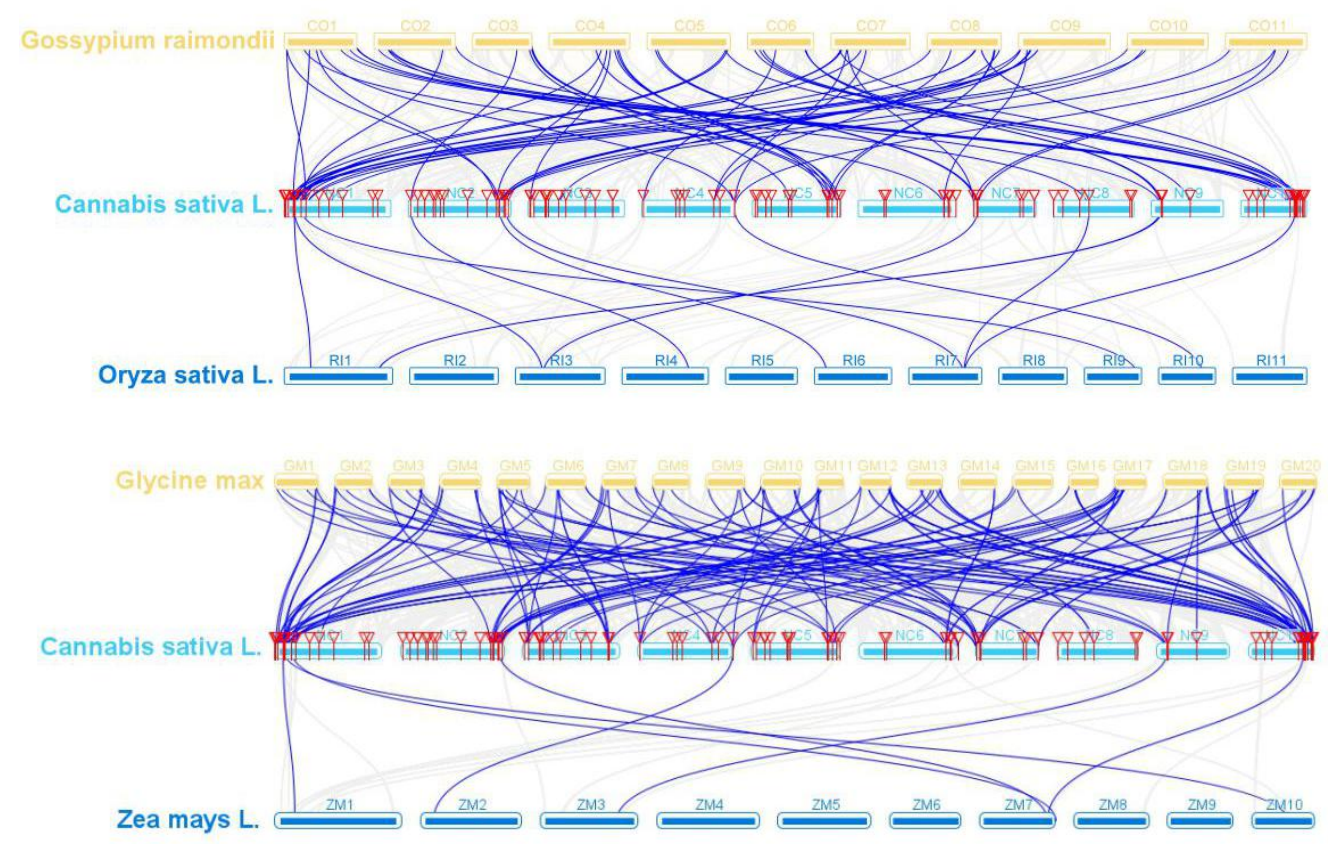

Figure 6. Synteny analyses between the MYB genes of Cannabis sativa L. and four representative plant species.

Gray lines in the background indicate collinear blocks within potato and other plant genomes, while blue lines highlight syntenic MYB gene pairs. 


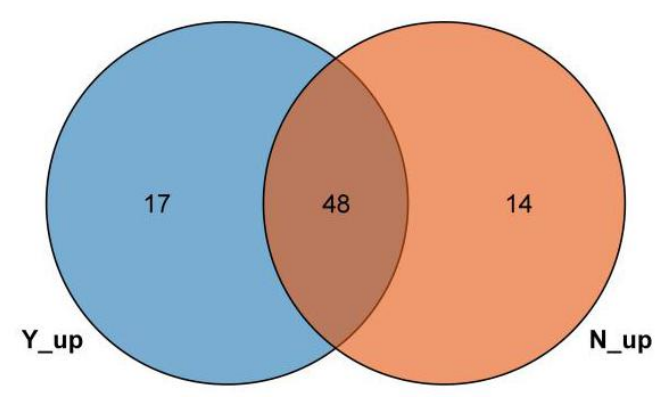

(a)

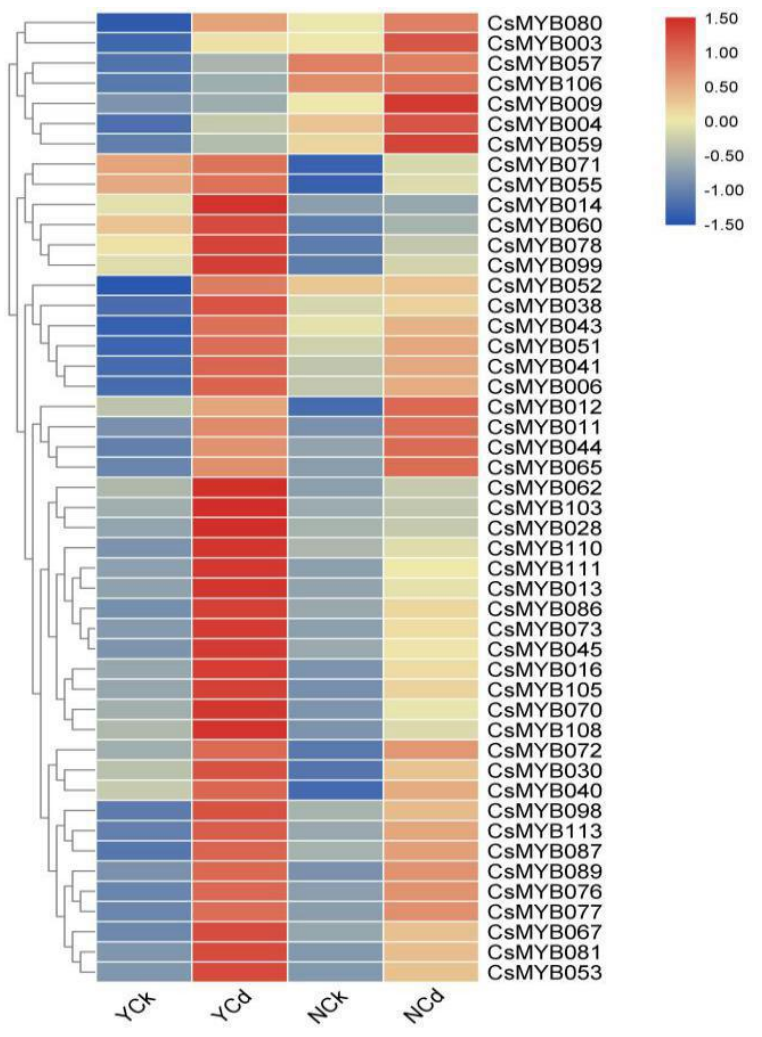

(b)

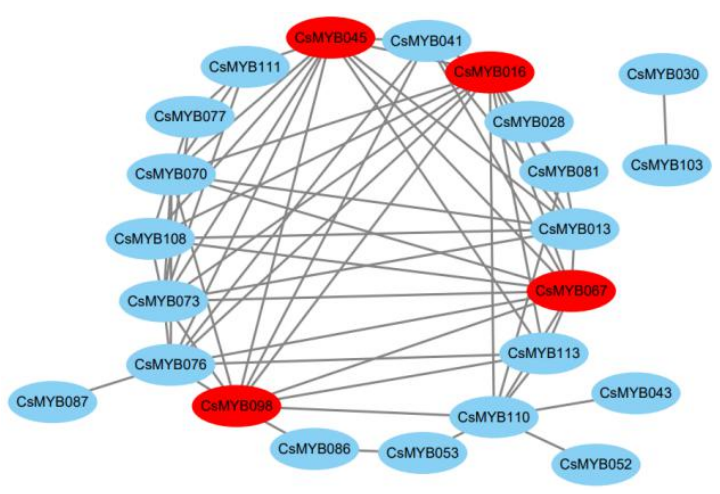

(c)

Figure 7. Association analysis of up-regulated MYB genes. (a) Venn diagram of up-regulated MYB base in two hemp varieties; (b) Heat map of MYB gene up-regulation in two hemp varieties, YCk mean Yunnan hemp No.1 with normal; Ycd mean Yunnan hemp No.1 with Cd-stress; (c) The correlation network of MYB up-regulated genes. 


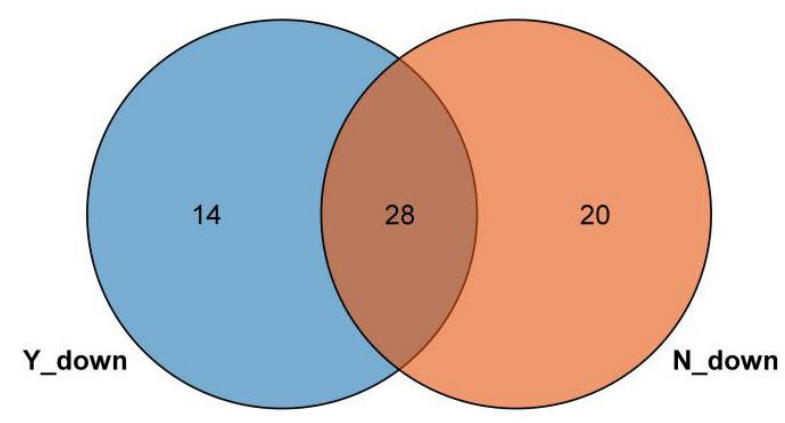

(a)

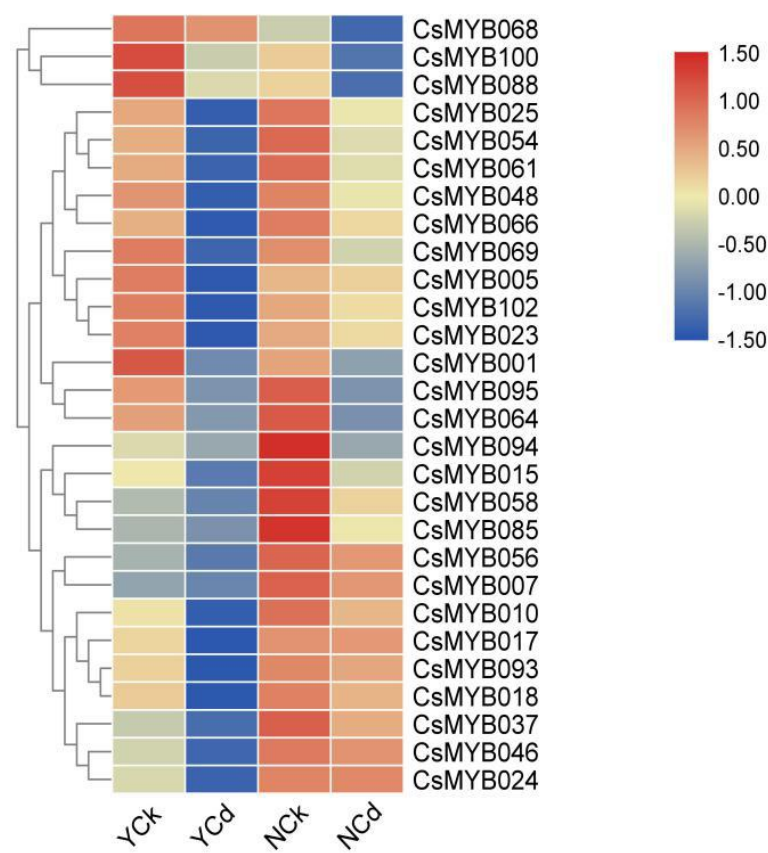

(b)

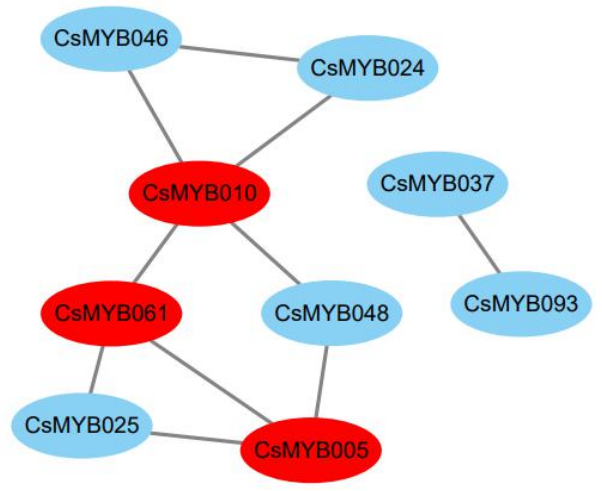

(c)

Figure 8. Association analysis of down-regulated MYB genes. (a) Venn diagram of down-regulated MYB base in two hemp varieties; (b) Heat map of MYB gene down-regulation in two hemp varieties, NCk mean Inner Mongolia small hemp with normal; Ncd mean Inner Mongolia small hemp with Cd-stress; (c) The correlation network of MYB up-regulated genes. 


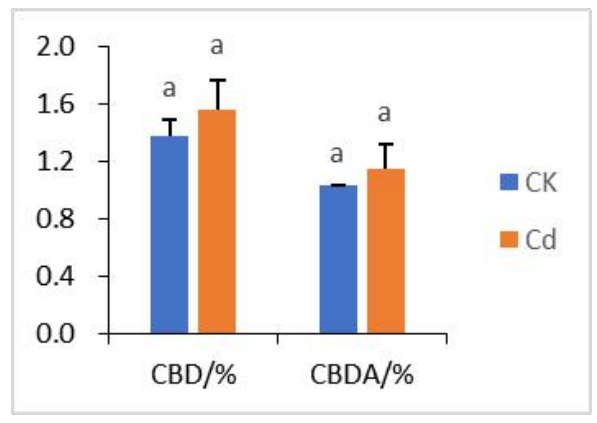

(a)

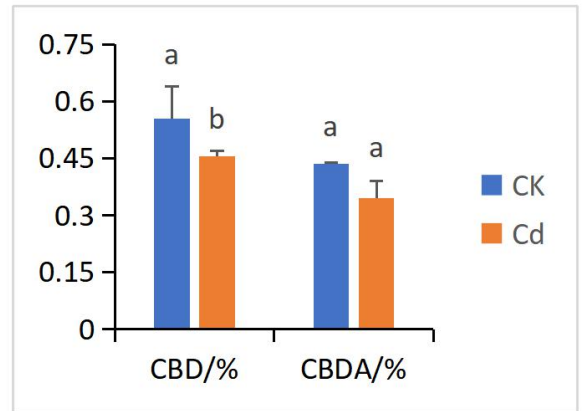

(b)

Figure 9. CBD and CBDA content in hemp, Lowercase letter(s) above the bars indicate significant differences (p $\leqslant 0.05$ ) among the treatments. (a) CBD and CBDA content in flowers; (b) CBD and CBDA content in leaves. 


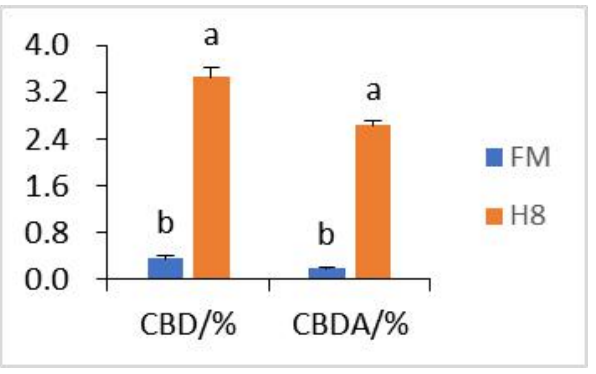

(a)

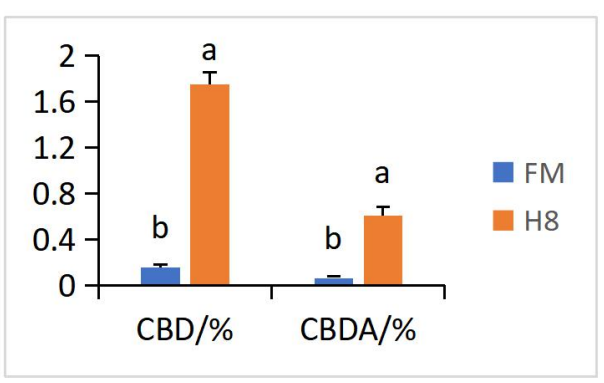

(b)

Figure 10. Hemp varieties with high and low CBD content, Lowercase letter(s) above the bars indicate significant differences $(\mathrm{p} \leqslant 0.05)$ among the treatments. (a) CBD and CBDA content in flowers; (b) CBD and CBDA content in leaves. 


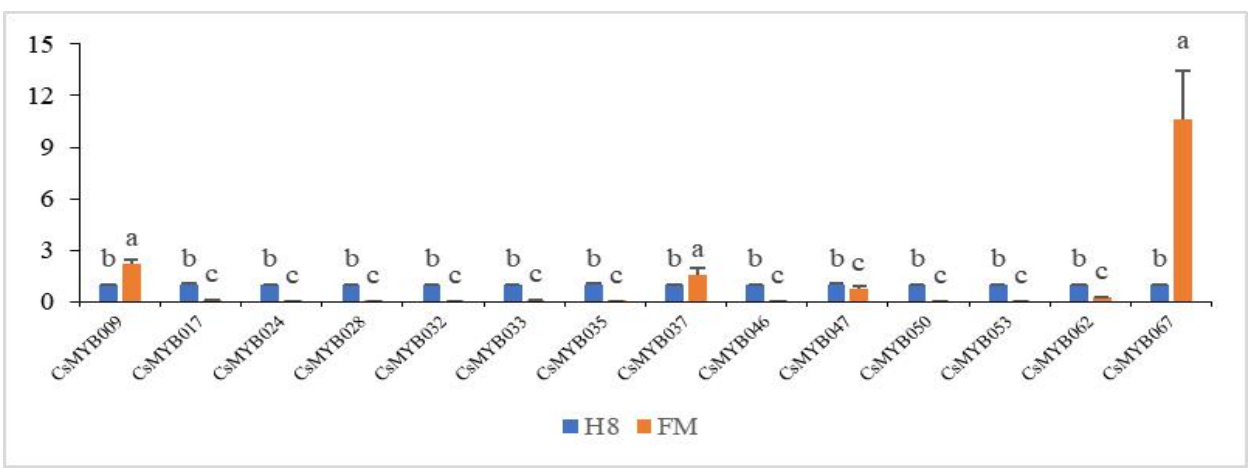

(a)

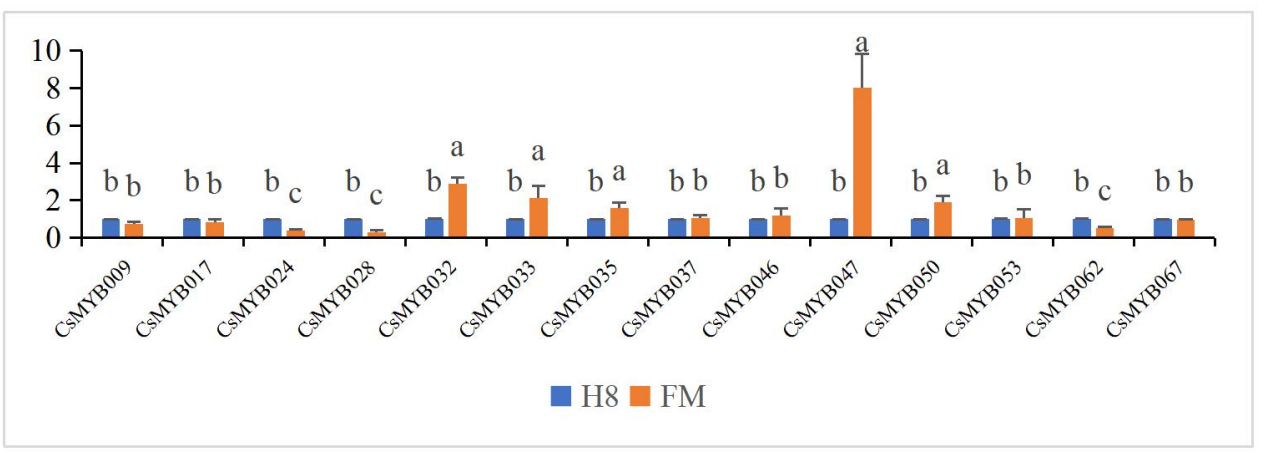

(b)

Figure 11. MYB gene expression in H8 and FM, Lowercase letter(s) above the bars indicate significant differences $(\mathrm{p} \leqslant 0.05)$ among the treatments. (A) MYB gene expression in flowers o

f different varieties. (B) MYB gene expression in leaves of different varieties. 


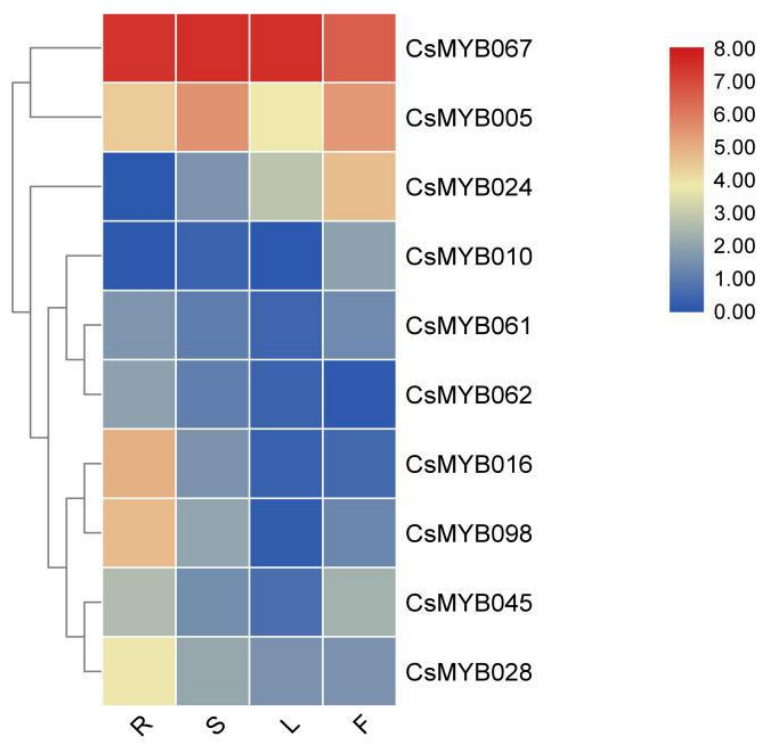

Figure 12. Tissue Specific Expression of Hemp MYB Gene, R means root, S means stem, L means leaf, F means female flower. 